\title{
Co-detection of GFP and dystrophin in skeletal muscle tissue sections
}

\author{
Kalliopi Liadaki ${ }^{1,2}$, Eric S. Luth², and Louis M. Kunkel ${ }^{1,2}$ \\ ${ }^{1}$ Howard Hughes Medical Institute, Chevy Chase, MD and ${ }^{2}$ Children's Hospital \\ Boston, MA, USA
}

BioTechniques 42:699-700 (June 2007)

doi $10.2144 / 000112494$

Duchenne muscular dystrophy (DMD) is characterized by progressive muscle degeneration that results from the absence of the cytoskeletal protein dystrophin. DMD cell-based therapy exploits the ability of various cell populations (muscle- and non-musclederived) to incorporate into diseased muscle and produce dystrophin. A common experimental approach involves the delivery of labeled donor cells and evaluation of their engraftment potential by quantifying expression of donor proteins in the muscles of recipient mice. Green fluorescent protein (GFP) is a unique protein with several positive features that make it an exceptional cell-tracking marker. GFP is easily detected, is genetically transmitted, diffuses rapidly in the cytoplasm of muscle fibers, and clearly delineates the morphology of the cell in which it is expressed. Most studies of DMD cell-based therapy used only GFP expression to measure donor cell engraftment into muscle, while some studies evaluated expression of GFP and dystrophin not in the same but in consecutive muscle sections $(1,2)$. Here, we report a method for simultaneous tracking of GFP fluorescence and dystrophin expression in the same muscle section.

GFP-labeled donor cells were isolated from the skeletal muscle of C57BL/6-Tg(ACTB-EGFP)1Osb/J mice as previously described (3). Recipient mice, C57B16Ros-5cv $\left(m d x^{5 c v}\right)$, show almost no background of revertant, dystrophin-positive fibers. GFP-labeled donor muscle cells $(80,000-150,000$ cells) were injected intramuscularly into the tibialis anterior (TA) muscle of $m d x^{5 c v}$ mice. After 6 weeks postinjection, host mice were euthanized, and excised TA muscles maldehyde solution (prepared fresh in sterile water) at $4^{\circ} \mathrm{C}$ in darkness and with continuous agitation using a rocker platform. The tissue was then soaked in $5 \%$ sucrose in phosphate-buffered saline (PBS; $0.22 \mu \mathrm{m}$ sterilized) for $6 \mathrm{~h}$ (or overnight) at $4{ }^{\circ} \mathrm{C}$, then in $20 \%$ sucrose in PBS $(0.22 \mu \mathrm{m}$ sterilized) overnight at $4{ }^{\circ} \mathrm{C}$ with continuous agitation for the optimal cryopreservation of muscle tissue. Muscle samples were embedded in optimal cutting temperature (OCT) compound, frozen by dipping for $30 \mathrm{~s}$ in liquid nitrogen-chilled isopentane, and stored at $-80^{\circ} \mathrm{C}$ until sectioning. This procedure preserves GFP expression in cells and muscle fibers without needing to transcardially perfuse the animal as previously suggested $(1,4-6)$.

For analysis of GFP and dystrophin expression, $10-\mu \mathrm{m}$-thick cross-sections were fixed overnight in $4 \%$ parafor-

were cut at $-25^{\circ} \mathrm{C}$ and were transferred onto Superfrost ${ }^{\circledR}$ Plus slides (VWR, West Chester, PA, USA) and immersed in cold PBS. Slides were examined under a Nikon Eclipse E1000 microscope, using a bandpass 535/50 filter, without mounting, at $100 \times$ magnification to identify sections with the highest number of GFPpositive fibers. Selected sections that displayed GFP-positive myofibers were fixed in methanol for $3 \mathrm{~min}$ at room temperature and then transferred to PBS for a maximum of 2 days before proceeding to dystrophin staining. If selected sections were not to be processed immediately, they were mounted in VECTASHIELD ${ }^{\circledR}$ (Vector Laboratories, Burlingame, CA, USA) media for fluorescence with or without a nuclear counterstaining by 4',6-diamidino-2-phenylindole (DAPI) and stored at $4^{\circ} \mathrm{C}$ for up to 1 week. Prior to dystrophin staining, sections were washed in PBS for 10 min with gentle agitation at room temperature in darkness before the coverslips were carefully removed.

For dystrophin staining, muscle sections were incubated in blocking buffer (PBS, $10 \%$ horse serum, $0.1 \%$ Triton ${ }^{\circledR}$ X-100) overnight at $4^{\circ} \mathrm{C}$. Sections were incubated with rabbit anti-mouse dystrophin polyclonal antibodies [6:10 antibody (7) diluted 1:1000 in blocking buffer or Abcam antibody (no. ab15277; Cambridge,
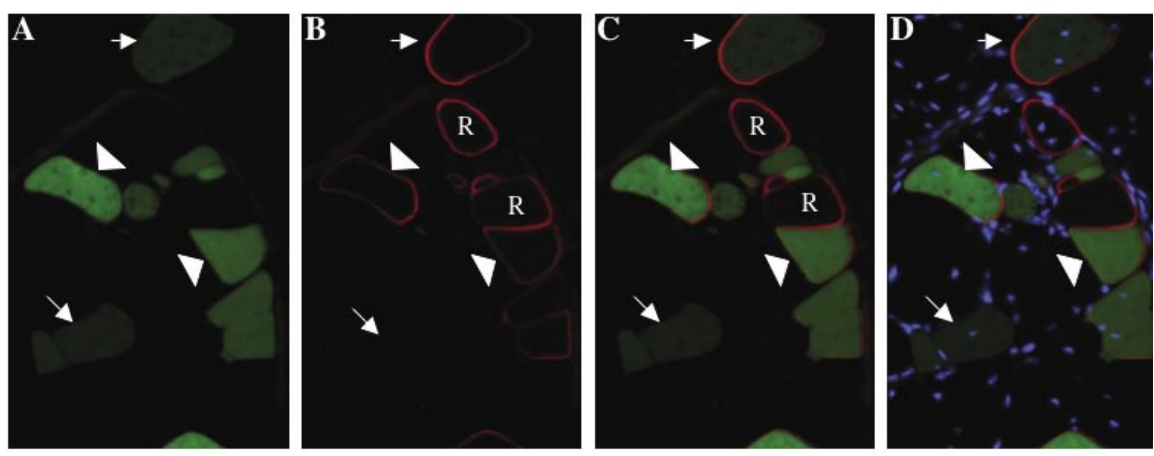

Figure 1. Co-detection of green fluorescent protein (GFP) and dystrophin in the same skeletal muscle section. (A) Expression of GFP, (B) dystrophin-Texas Red, (C) GFP and dystrophin, and (D) GFP/dystrophin/4',6-diamidino-2-phenylindole (DAPI) in tibialis anterior muscle of recipient mice following intramuscular injections of GFP-positive muscle cells. For detection of dystrophin, the rabbit polyclonal 6:10 antibody (7) was used. Panels A-D were acquired using a 20× objective corresponding to $200 \times$ overall magnification. As opposed to autofluorescence, true GFP expression is detectable over background fluorescence levels. For example, muscle fibers are fluorescent only when using the fluorescein filter, but not the rhodamine filter. There is variation in the level of GFP expression, with highGFP-expressing fibers (arrowheads) and low-GFP-expressing fibers (arrows). The presence of revertant dystrophin-expressing fibers, which do not express GFP, is marked with R. 


\section{Benchmarks}

MA, USA) diluted 1:100 in blocking buffer] overnight at $4^{\circ} \mathrm{C}$. Sections were washed three times for $10 \mathrm{~min}$ each with $\mathrm{PBS} / 0.1 \%$ Triton $\mathrm{X}-100$ at room temperature, and subsequently incubated with Texas Red-conjugated anti-rabbit immunoglobulin $\mathrm{G}$ (IgG; Jackson Immunoresearch, West Grove, PA, USA) diluted 1:100 in blocking buffer overnight at $4^{\circ} \mathrm{C}$. After three washes of 10 min each with PBS/0.1\% Triton X100 at room temperature, sections were coverslipped with mounting medium with or without DAPI.

Sections were examined under a Nikon Eclipse E1000 microscope using the following emission filters: bandpass 535/50 for GFP detection; bandpass 605/55 for Texas Red detection; and bandpass $460 / 50$ for DAPI detection. Images were collected from the same microscopic field with separate filters for GFP, Texas Red, and DAPI signals using a charge-coupled device (CCD) camera (Diagnostic Instruments, Sterling Heights, MI, USA). Signals were overlaid using Openlab software (Improvision, Lexington, MA, USA). Specificity of the signal observed on GFP-labeled fibers (as opposed to muscle fiber autofluorescence) was assayed by ensuring that the signal could be detected only with the fluorescein filter and not with the rhodamine filter. In addition, the use of the specific narrow bandpass filter for GFP fluorescence allows to clearly distinguish between true GFP fluorescence and autofluorescence; true GFPpositive fibers exhibit bright, diffuse, and pure-green fluorescence, while autofluorescent fibers are usually less fluorescent with common marginal enhancement not observed in GFPpositive muscle fibers. The level of GFP expression varies between fibers, depending on the number of donor nuclei fused and the distance that GFP diffuses (Figure 1, arrows and arrowheads). In addition, GFP-negative/ dystrophin-positive revertant fibers are easily identified (Figure 1, labeled as $\mathrm{R})$. The percentage of GFP-positive/ dystrophin-positive fibers in various sections ranges from $25 \%$ to $54 \%$. These numbers are greater compared with previous studies, which evaluated GFP and dystrophin expression on consecutive sections $(1,2)$. A large percentage
$(>50 \%)$ of GFP-positive fibers were dystrophin-negative. This finding was expected, since the expression domain of membrane-bound dystrophin differs from that of cytosolic GFP. It is widely accepted that dystrophin is present only within a limited distance from the nucleus containing the activated gene, forming the so-called nuclear domain (8). Alternatively, the fusion of donor nuclei to the recipient muscle fibers might not contribute to dystrophin expression in the host/dystrophic environment (9). This technique can also be applied when donor cells express GFP as a result of viral transduction. In these cases it would be preferable to label donor cells with a virus (e.g., retrovirus) that allows for a persistent GFP expression, even after cells proliferate in the host muscle. The use of certain viruses that transiently label donor cells will result in a loss of GFP expression after cell proliferation, thus creating false-negative results since the cells might express only dystrophin, but not GFP.

In conclusion, we report a method for co-detection of GFP and dystrophin in the same muscle section. GFP expression in recipient muscle is suggestive but not sufficient to evaluate the therapeutic potential of donor cells, unless it is demonstrated that donor cells following fusion with pre-existing myofibers also express dystrophin. Furthermore, it is necessary to distinguish between donor cell-derived dystrophin and revertant dystrophin to evaluate donor cell efficiency, even in cases when models with few revertant fibers (like $m d x^{5 c v}$ ) are used. This analysis should be performed on the same muscle section, especially in cases when the material of interest is rare to acquire. This method will be of significant use in the evaluation of the therapeutic potential of different cell populations for treating DMD.

\section{ACKNOWLEDGMENTS}

This work was supported by a grant from the Muscular Dystrophy Association and the generous contribution of the Bernard F. and Alva B. Gimbel Foundation. L.M.K. is an investigator with the Howard Hughes Medical Institute.

\section{COMPETING INTERESTS STATEMENT}

The authors declare no competing interests.

\section{REFERENCES}

1. Chretien, F., P.A. Dreyfus, C. Christov, P. Caramelle, J. Lagrange, B. Chazaud, and R.K. Gherardi. 2005. In vivo fusion of circulating fluorescent cells with dystrophin-deficient myofibers results in extensive sarcoplasmic fluorescence expression but limited dystrophin sarcolemmal expression. Am. J. Path. 166:1741-1748.

2.Fukada, S., Y. Miyagoe-Suzuki, H. Tsukihara, K. Yuasa, S. Higuchi, S. Ono, K. Tsujikawa, S. Takeda, and H. Yamamoto. 2002. Muscle regeneration by reconstitution with bone marrow or fetal liver cells from green fluorescent protein-gene transgenic mice. J. Cell Sci. 115:1285-1293.

3. Montanaro, F., K. Liadaki, J. Schienda, A. Flint, E. Gussoni, and L.M. Kunkel. 2004. Demystifying SP cell purification: viability, yield, and phenotype are defined by isolation parameters. Exp. Cell Res. 298:144-154.

4.Brazelton, T.R. and H.M. Blau. 2005 Optimizing techniques for tracking transplanted stem cells in vivo. Stem Cells 23:1251-1265.

5. Wernig, G., V. Janzen, R. Schafer, M. Zweyer, U. Knauf, O. Hoegemeier, R.R. Mundegar, S. Garbe, et al. 2005. The vast majority of bone-marrow-derived cells integrated into mdx muscle fibers are silent despite long-term engraftment. Proc. Natl. Acad. Sci. USA 102:11852-11857.

6.Jackson, K.A., D.S. Snyder, and M.A. Goodell. 2004. Skeletal muscle fiber-specific green autofluorescence: potential for stem cell engraftment artifacts. Stem Cells 22:180187.

7.Lidov, H.G., T.J. Byers, S.C. Watkins, and L.M. Kunkel. 1990. Localization of dystrophin to postsynaptic regions of central nervous system cortical neurons. Nature 348:725-728.

8.Pavlath, G.K., K. Rich, S.G. Webster, and H.M. Blau. 1989. Localization of muscle gene products in nuclear domains. Nature 337:570-573.

9. Gussoni, E., H.M. Blau, and L.M. Kunkel. 1997. The fate of individual myoblasts after transplanatation into muscles of DMD patients. Nat. Med. 3:970-977.

Received 12 March 2007; accepted 26 April 2007.

Address correspondence to Louis $M$. Kunkel, Children's Hospital, 320 Longwood Avenue, Enders 5, Boston, MA 02115, USA. e-mail:kunkel@enders.tch.harvard.edu

To purchase reprints of this article, contact: Reprints@BioTechniques.com 